\title{
PARÂMETROS AMBIENTAIS E MATERIAIS QUE AFETAM A PENETRAÇÃO DE CLORETOS EM ESTRUTURAS DE CONCRETO - ESTUDO DE CASO DA ILHA DOS ARVOREDOS
}

\section{Environmental and material parameters that affect chloride ingress in reinforced concrete structures - Case study of Arvoredos Island}

\author{
Carlos Eduardo Tino Balestra', Maryangela Geimba de Lima², \\ Ronaldo A. Medeiros-Junior ${ }^{3}$, Ana Julia Alves Egg Monteiro ${ }^{4}$
}

Recebido em 31 de agosto de 2016; recebido para revisão em 06 de janeiro de 2017; aceito em 07 de março de 2017; disponível on-line em 29 de março de 2017.

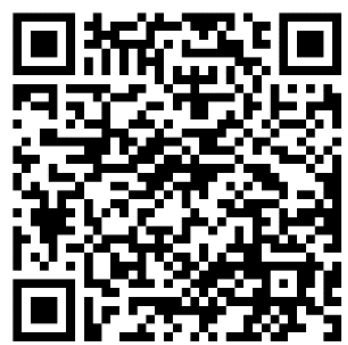

PALAVRAS CHAVE:

Corrosão;

Cloretos;

Durabilidade;

Armaduras;

Ambiente marinho.

\section{KEYWORDS:}

Corrosion;

Chlorides;

Durability;

Reinforcement;

Marine environment.

RESUMO: A corrosão das armaduras induzida por cloretos é um dos principais problemas relacionados à degradação de estruturas de concreto armado em ambiente marinho. Desta forma, este artigo apresenta uma compilação e discussão dos principais parâmetros ambientais e materiais que influenciam na penetração de cloretos em estruturas de concreto presentes neste ambiente. Além disso, como estudo de caso, ensaios de espectroscopia de fluorescência de Raios-X em amostras de concreto foram realizados em pontos de algumas estruturas presentes em diferentes zonas de agressividade marinha. As conclusões deste trabalho mostram que a degradação de uma estrutura pode ser evitada desde a etapa de projeto e que há um grande número de variáveis ambientais e materiais que afetam o processo. Esse trabalho contribui para a evolução do conhecimento sobre estruturas que sofrem degradação natural, expostas ao ambiente marinho. Os ensaios de espectroscopia de fluorescência de Raios-X mostraram que, sob uma perspectiva da corrosão, a zona de variação de maré é a mais agressiva às estruturas de concreto armado. Os ciclos de molhagem e secagem aos quais a zona de variação de maré está sujeita contribuem para acelerar a penetração dos cloretos no concreto. Portanto, de acordo com o tipo de zona marinha onde as estruturas podem estar presentes, o nível de agressividade quanto à corrosão induzida por cloretos é variável.

ABSTRACT: The reinforcement corrosion induced by chlorides is one of the main problems related to the degradation of reinforced concrete structures in marine environment. Thus, this paper presents a compilation and discussion of key environmental and materials parameters that influence the chloride penetration in concrete structures present in this environment. Furthermore, as a case study, Fluorescence Spectroscopy X-ray testing of concrete samples were made in points of structures presents in different marine aggressive zones. The conclusions of this study show that degradation of a structure can be prevented from the design stage and that there are a large number of environmental and materials variables involved. This paper contributes to knowledge evolution about structures with natural degradation that are exposed to the marine environment. The X-ray fluorescence spectroscopy experiments showed that, from a perspective of corrosion, tidal zone is the most aggressive zone to reinforced concrete structures. The wetting and drying cycles contribute to accelerate the penetration of the chlorides into the concrete. Therefore, according to the type of marine zone where structures may be present, the level of aggressiveness for chloride-induced corrosion is variable.

* Contato com os autores:

${ }^{1}$ e-mail: carlosbalestra@utfpr.edu.br (C. E. T. Balestra)

Professor mestre do Departamento de Engenharia Civil da Universidade Tecnológica Federal do Paraná.

2e-mail: magdlima@ita.br ( M. G. Lima)

Professora Doutora da Divisão de Engenharia Civil do Instituto Tecnológico de Aeronáutica.

${ }^{3}$ e-mail: ronaldodemedeirosjr@yahoo.com.br (R. A. Medeiros-Junior)

Professor Doutor do Departamento de Construção Civil da Universidade Federal do Paraná.

2e-mail: anajulia_92@hotmail.com (A. J. A. E. Monteiro)

Discente do Programa de Pós-Graduação em Engenharia de Construção Civil da Universidade Federal do Paraná. 


\section{INTRODUÇÃO}

A degradação de estruturas de concreto armado em ambiente marinho é objeto de estudo por pesquisadores de durabilidade e patologia das estruturas. Sabe-se que a corrosão de armaduras induzidas por cloretos é um dos grandes problemas do ambiente marinho no quesito degradação. Quando a concentração de cloretos na armadura supera um valor crítico, a camada de proteção das armaduras é destruída e o processo de corrosão é iniciado. Nesse processo são formados produtos que devido à sua característica expansiva, podem iniciar um quadro de fissuração no concreto ou até mesmo lascamento. Além das manifestações patológicas que podem ser originadas no concreto, há também a possibilidade de redução da seção transversal da armadura, reduzindo consideravelmente a capacidade portante do elemento estrutural, principalmente pela característica de ataque desses cloretos, por pites. Além disso, a estrutura também está à deriva da agressividade do meio, e de impactos físicos como o impacto das ondas.

Sob uma perspectiva da infraestrutura marítima, cabe salientar seu papel fundamental na economia de diversas nações. Tomando em específico o caso do Brasil, um país de extensa costa litorânea, mais de $95 \%$ das movimentações de exportação e importação de produtos passam pela infraestrutura portuária nacional (Da Costa et al., 2013), sendo muitas dessas infraestruturas existentes executadas totalmente ou parcialmente em concreto armado. Além disso, é possível citar ainda como exemplos de construções marítimas as plataformas fixas destinadas à extração de petróleo, pontes, píeres e as expansões marítimas que vêm sendo construídas para alocar obras de infraestrutura nas últimas décadas como, por exemplo, os aeroportos de Kansai e Chúbu no Japão. Isto demonstra que há um número expressivo de estruturas totalmente ou parcialmente sujeitas aos efeitos degradantes do ambiente marinho.

Portanto, esse artigo se justifica pelo fornecimento de informações sobre o ingresso de cloretos em estruturas que sofreram degradação natural, visto que o Brasil, com sua grande extensão litorânea, possui muitas estruturas de concreto na zona costeira. O entendimento acerca dos mecanismos e das variáveis atuantes no processo de penetração de cloretos auxilia na geração de novas tecnologias que consigam postergar ou evitar a penetração desses agentes agressivos nas estruturas de concreto armado, além da diminuição dos custos de manutenção e do aumento da durabilidade da estrutura.

\section{OBJETIVO}

O presente artigo tem como objetivo apresentar uma revisão dos principais parâmetros ambientais e materiais que influenciam na penetração de cloretos em estruturas de concreto presentes em ambiente marinho. Os principais parâmetros abordados são:

- Ambientais: salinidade atmosférica e da água do mar, ventos, umidade relativa, temperatura, chuva, carbonatação, presença de espécies químicas na água do mar (sulfatos e magnésio) e efeitos das mudanças climáticas;

- Materiais: tipo de cimento, relação água/cimento $(\mathrm{a} / \mathrm{c})$, regime de cura do concreto e adições.

Como parte complementar dos objetivos, será realizado um estudo de caso (Ilha dos Arvoredos). Portanto, a discussão a respeito das diferentes concentrações de cloretos nas diferentes zonas de agressividade marinha em uma estrutura real de concreto armado também é objetivo deste artigo.

\section{PROCESSO CORROSIVO DAS ESTUTURAS DE CONCRETO ARMADO EM AMBIENTE MARINHO}

O caráter alcalino do $\mathrm{pH}$ da solução presente nos poros do concreto fornece condições estáveis à formação de um filme passivante que reveste as armaduras protegendo-as frente à corrosão. Entretanto, em ambiente marinho, os 
cloretos advindos da água do mar penetram a camada de cobrimento, através da rede de poros, e chegam à região onde as armaduras estão presentes. Quando a concentração destes supera um valor crítico, o filme passivante é destruído localmente e o processo corrosivo das armaduras encontra condições para seu estabelecimento e propagação, como demonstrado pelo modelo proposto por Tuutti (1982). Como consequência deste processo, o metal das armaduras é consumido e são formados produtos de corrosão expansivos levando à degradação das estruturas de concreto armado (François et al., 2013; Zhu et al., 2013; Hanet al., 2014; Khan et al., 2014).

Em primeira instância, os produtos de corrosão, ao serem formados, se depositam na superfície periférica das barras, e, devido ao seu caráter expansivo, ocupam um volume até oito vezes superior ao metal consumido, exercendo tensões radiais ao eixo das armaduras que levam à fissuração e posteriormente ao lascamento da camada de cobrimento. Em segundo lugar, o consumo do metal durante o processo corrosivo leva a uma redução contínua da seção transversal das armaduras; consequentemente, há uma redução da área de aço destinada a resistir a esforços de tração e uma diminuição progressiva da capacidade resistente do elemento estrutural executado em concreto armado. Como o processo corrosivo é evolutivo e agrava-se em seu decurso, níveis elevados de corrosão podem esgotar a capacidade resistente de uma estrutura levando a mesma ao colapso (Apostolopoulos et al., 2013; Hanet al., 2014 Medeiros-Junior et al., 2015a).

A ação dos cloretos conduz a um processo de corrosão denominado corrosão por pites, sendo estes caracterizados por pontos bem definidos na superfície das armaduras que se aprofundam à medida que o processo corrosivo avança (Cascudo, 1998). A Figura 1 apresenta a microscopia de uma barra com aumento de $260 x$ onde é possível observar a presença de diversos pites.

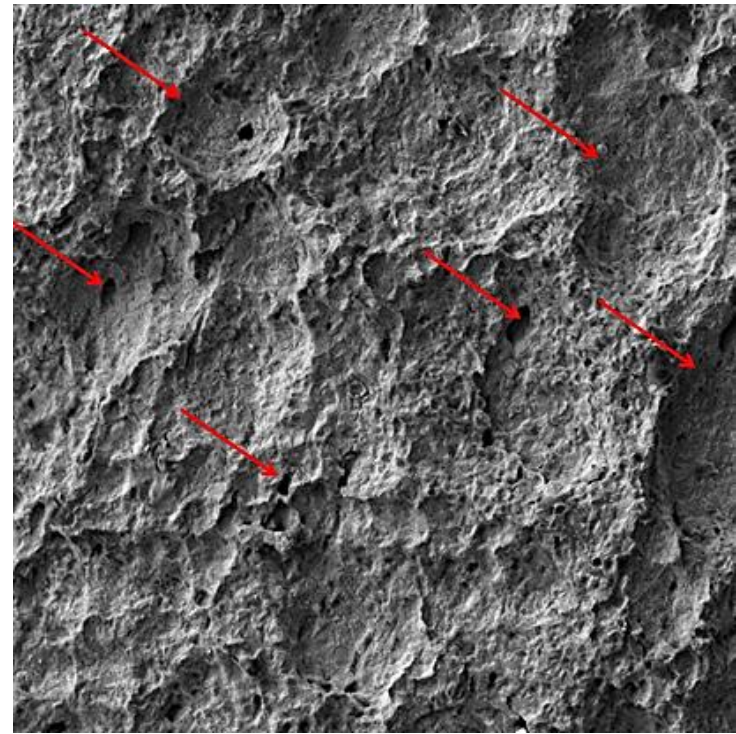

FIGURA 1: Microscopia Eletrônica de Varredura mostrando a presença de pites em uma armadura com aumento de 260x.

FONTE: AUTORES.

Os pites são capazes de gerar pequenas variações de massa nas armaduras, porém com danos significativos à sua seção transversal, reduzindo de forma notável as propriedades mecânicas das barras quando submetidas à tração (Balestra, 2013). Zhu et al. (2014) observaram que os danos causados por pites acabam gerando excentricidades entre a posição dos eixos de seções corroídas e não corroídas das barras, sendo que, a medida que os pites aprofundando-se, maiores são estas excentricidades. Consequentemente, quanto maior as excentricidades, menor os valores pertinentes às propriedades mecânicas das armaduras quando submetidas à tração.

Entre todos os ambientes onde as estruturas de concreto podem estar presentes, o ambiente marinho apresenta-se como um dos mais agressivos, uma vez que as estruturas podem estar sujeitas a ataques físicos, pela ação mecânica do impacto das ondas e partículas em suspensão na água, e químicos, em vista da presença de espécies químicas na água do mar que são capazes de penetrar através da rede de poros do concreto e reagir com os produtos de hidratação do cimento, levando à degradação do concreto, ou das armaduras, por meio da corrosão (Broomfield, 2007; Mehta e Monteiro, 2008; Angst et al., 2009; Medeiros et al., 2013). 
Uma estrutura de concreto em ambiente marinho pode estar sujeita a diferentes níveis de ataque dependendo da zona de agressividade no qual se encontra. Neste caso, o ambiente marinho pode ser dividido em quatro zonas: atmosfera marinha, respingo, variação de maré e submersa. $\mathrm{Na}$ primeira zona, embora não esteja em contato com a água do mar, a estrutura sofre degradação por conta do aerossol marinho composto por sais e gotículas de água que são carregados pela ação dos ventos provenientes do mar. $\mathrm{Na}$ zona de respingos as estruturas recebem os respingos das ondas do mar, e na zona de variação de maré a estrutura está situada entre os níveis máximos e mínimos alcançados pela linha de água; nesta zona, a estrutura está sujeita a ciclos de molhagem e secagem que favorecem condições para acelerar o ingresso de cloretos no concreto. $\mathrm{Na}$ zona submersa, a estrutura permanece continuamente submersa (Mehta e Monteiro, 2008). Segundo Safehian et al. (2013) a zona de variação de maré geralmente é a mais agressiva quanto à penetração de cloretos, seguida pela zona de respingos.

Samarakoon e Ratnayake (2013) alertam que o monitoramento sistemático e contínuo da penetração de cloretos em estruturas de concreto em ambiente marinho não é realizado em muitos casos, onde por vezes, admite-se como premissa de projeto que o concreto utilizado como camada de cobrimento nas obras é unicamente e suficientemente capaz de prover resistência ao ingresso dos cloretos e proteção às armaduras. Tal discussão sugere que muitas vezes as medidas que visem à proteção das estruturas de concreto armado em ambiente marinho são desprezadas desde a etapa de concepção de um projeto.

Assim, o conhecimento referente aos parâmetros que afetam a penetração de cloretos em estruturas neste ambiente é uma ferramenta importante para prover informações desde a etapa de projeto. Com esse conhecimento prévio, é possível estimar o tempo necessário para que os cloretos atinjam a região das armaduras e iniciem o processo corrosivo através de diferentes modelos matemáticos disponíveis na literatura
(Li et al., 2015; Wu et al., 2016). Isso sugere um aspecto de dimensionamento da estrutura com um conceito de durabilidade incluso, diferentemente de um dimensionamento apenas relacionado com a resistência mecânica do material.

\section{FATORES AMBIENTAIS QUE INFLUENCIAM NA AGRESSIVIDADE ÀS ESTRUTURAS DE CONCRETO ARMADO PRESENTES EM AMBIENTE MARINHO}

\subsection{SALINIDADE ATMOSFÉRICA E DA ÁGUA DO MAR, E INFLUÊNCIA DOS VENTOS}

A salinidade atmosférica tem sido objeto de diversas pesquisas internacionais (Mustafa e Yusof 1994; Corvo et al., 1995; Morcillo et al., 2000; Lee e Moon, 2006 e Meira et al., 2006). Nesta vertente de estudos, os ventos ganham destaque por dois motivos principais. Em primeiro lugar como responsáveis na geração das ondas do mar, propiciando a formação do aerossol marinho, e em segundo lugar, por atuarem como agente transportador do aerossol para o continente, possibilitando a deposição de sais sobre a superfície das estruturas de concreto presentes em zona de atmosfera marinha.

Segundo Morcillo et al. (2000), as partículas salinas presentes no aerossol marinho com diâmetro superior à $10 \mu \mathrm{m}$ permanecem em suspensão na atmosfera por curtos períodos de tempo, sendo depositadas a curtas distâncias a partir da costa pelo efeito gravitacional. Partículas menores, com diâmetro inferior à $10 \mu \mathrm{m}$, podem ser carregadas pelo vento a maiores distâncias, atingindo estruturas mais afastadas da costa. Meira et al. (2007) pontuam que a maior influência da deposição de partículas salinas ocorre até uma distância de $200 \mathrm{~m}$ em relação à costa. Além disso, a ocorrência de eventos climáticos extremos de curta duração como as tempestades, com fortes ventos e grande agitação do mar, é responsável por carregar grandes quantidades de sais, que são posteriormente depositados sobre a superfície das estruturas em curtos períodos de tempo (Morcillo et al., 2000). Em estudos similares, Pontes (2006) e 
Romano (2009) também identificaram que a agressividade por cloretos é mais importante nas primeiras faixas de terra, principalmente nos primeiros 400 metros, segundo Pontes (2006), e entre 100 a 200m, de acordo com Romano (2009). Para a região do mediterrâneo, Jaegerman (1990) observou que nos primeiros 400 metros a partir da costa, há uma redução acentuada da concentração de cloretos no aerossol marinho.

De uma forma geral, a salinidade atmosférica é determinada através dos aparatos de vela úmida normalizados pela ASTM G140. Neste caso, uma solução de glicerina é utilizada para o monitoramento periódico da deposição de sais presentes na atmosfera.

Os resultados obtidos a partir da exposição de velas úmidas mostram que há um aumento notável da taxa de deposição de sais nos aparatos à medida que a velocidade dos ventos supera 3m/s (Morcillo et al., 2000; Meira et al. 2006 e 2007). Neste sentido, Lee e Moon (2006) modelaram o decréscimo da taxa de deposição de sais à medida que a distância em relação ao mar aumenta, obtendo uma equação exponencial com coeficientes de ajuste dependentes das condições locais.

Sob a perspectiva da influência da salinidade e temperatura da água do mar na penetração de cloretos, Lindvall (2007) estudou corpos de prova de concreto submersos por um ano em diferentes partes do mundo, sendo posteriormente analisados quanto à penetração de cloretos. Os resultados indicaram que quanto maior a salinidade, maior o ingresso de cloretos nos corpos de prova.

\subsection{UMIDADE RELATIVA, TEMPERATURA E CHUVA}

A umidade relativa e a temperatura apresentam importância principalmente sob os ciclos de molhagem e secagem que ocorrem nas camadas mais superficiais do concreto parcialmente saturado (Castro et al., 2001).

A umidade relativa do ar influencia as propriedades do concreto, tanto na fase de cura e endurecimento, quanto nas suas condições e desempenho quando colocado em serviço. Altos níveis de umidade acabam proporcionando um gradiente entre a umidade presente nas camadas de concreto mais próximas à superfície e as camadas mais internas do concreto. Isto favorece a penetração de cloretos para o interior do material, por mecanismo de absorção capilar, que ocorre principalmente nas camadas mais próximas à superfície, e mecanismo de difusão nas camadas mais internas do concreto. A delimitação entre as regiões onde ocorrem estes mecanismos é caracterizada pela ocorrência de um máximo (pico) na concentração de cloretos (Castro et al., 2001; Meira et al., 2007; Arya et al., 2014). A condição de umidade também governa o grau de saturação dos poros do concreto e fornece um efeito decisivo nos caminhos disponíveis para a difusão dos íons cloretos. Segundo o Comité Euro-International Du Béton (1992), há uma faixa ideal de umidade relativa que favorece $\mathrm{o}$ ingresso de cloretos no concreto, e que proporciona um alto risco de corrosão, situada em torno de $85 \%$.

A respeito da temperatura, Song et al. (2008) e Yuan et al. (2009) pontuam que os efeitos desta acabam por acelerar o movimento iônico dos cloretos no concreto. De fato, Oh e Jang (2007), por meio de modelos matemáticos, concluíram que o aumento da temperatura acaba por aumentar a mobilidade iônica dos mesmos nos poros do concreto. Mazer (2009) verificou que em temperaturas elevadas há um aumento no valor do coeficiente de difusão de cloretos. A temperatura também interfere nos parâmetros de corrosão, tais como potencial de corrosão, resistividade e taxas de corrosão. Por outro lado, seus efeitos durante o processo de cura podem reduzir os coeficientes de difusão devido a melhora na microestrutura, como, por exemplo, quando a cura à vapor é utilizada.

Dois efeitos são descritos na literatura no tocante a chuva. O primeiro trata da conversão de partículas salinas depositadas sobre a superfície das estruturas de concreto em soluções salinas que, por mecanismos de absorção capilar, acabam transportando os íons agressivos para o interior do concreto, ao passo que o segundo efeito trata da 
lavagem das superfícies de concreto, geralmente relacionadas às chuvas dirigidas que acabam carregando as partículas salinas a partir da superfície das estruturas de concreto (Meira et al., 2006; Medeiros-Junior et al., 2015b).

\subsection{CARBONATAÇÃO, SULFATOS E MAGNÉSIO}

Em primeiro lugar, antes de tratar dos efeitos da carbonatação, é importante ressaltar as interações que ocorrem entre os cloretos e a matriz cimentícia, a medida que estes atravessam a camada de cobrimento do concreto. Neste sentido, ao adentrarem o concreto, uma parte dos cloretos é capturada pelas fases aluminato encontradas na pasta de cimento formando sais (Sal de Friedel e Sal de Kuzel); esse processo é denominado fixação química dos cloretos. Esta fixação implica em uma redução da quantidade de cloretos passíveis de se movimentar através da rede de poros do concreto, denominados cloretos livres, e chegar à região das armaduras propiciando condições para o estabelecimento do processo corrosivo (Yuan et al., 2009).

A carbonatação é passível de ocorrer no ambiente marinho em regiões costeiras urbanizadas e industriais, e seus efeitos são citados em pesquisas recentes de laboratório, principalmente para tentar entender o efeito da liberação de cloretos previamente fixados quimicamente na microestrutura do concreto. Neste sentido, a carbonatação do concreto acaba promovendo a liberação de cloretos fixados junto às fases aluminato encontradas na pasta de cimento e, consequentemente, aumentam a quantidade de cloretos livres em solução que são passiveis de se movimentar através da rede de poros e chegar até a região das armaduras, desencadeando o processo corrosivo. Todavia, conclusões definitivas a respeito deste fenômeno ainda necessitam de maiores estudos (Backus et al., 2013; Kuosa et al., 2014; Liu et al., 2014; Saillio et al., 2014).

Com relação aos sulfatos, Maes e De Belie (2014) e Wang et al. (2013) pontuam primeiro que na água do mar, além da presença de cloretos, há a presença de sulfatos, em geral, associados ao sódio, onde, neste caso, o ataque pelo sulfato de sódio resultará em reações com as fases aluminato da pasta de cimento levando à formação de etringita, havendo variações de ordem volumétrica que acabam favorecendo à fissuração do concreto. Com relação ao magnésio, Maes e De Belie (2014) pontuam que a presença deste pode acarretar em reações de troca catiônica levando a formação de produtos frágeis com características não cimentícias. Ambos os processos favorecem a penetração de cloretos no concreto devido à formação de fissuras no material.

\subsection{EFEITOS DAS MUDANÇAS CLIMÁTICAS}

$\mathrm{Na}$ literatura há dificuldade de encontrar estudos que tratem dos efeitos das mudanças climáticas sobre a durabilidade das estruturas de concreto. De uma forma geral, os trabalhos tratam da aplicação de modelos de previsão de vida útil de estruturas de concreto sob diferentes cenários. Neste sentido, destaque é dados aos trabalhos de Bastigas-Arteaga et al. (2010) e Medeiros-Junior et al. (2015b).

O primeiro conduziu um estudo a respeito da influência do aquecimento global sobre o ingresso de cloretos no concreto. Os resultados obtidos a partir de simulações conduzidas em modelos que contemplam efeitos de convecção, idade do concreto, temperatura e umidade sob três diferentes cenários mostram que as mudanças climáticas podem levar a uma considerável redução da vida útil de estruturas de concreto, a partir de uma projeção de 100 anos. De fato, o trabalho de Medeiros-Junior et al. (2015b) concorda que os efeitos das mudanças climáticas têm um impacto considerável sobre a vida útil das estruturas de concreto. Seus resultados indicaram que as mudanças de temperatura e umidade relativa previstas para o ano de 2100 podem levar a uma redução da ordem de 10 anos na vida útil de estruturas de concreto.

\section{FATORES MATERIAIS QUE INFLUENCIAM NA AGRESSIVIDADE ÀS ESTRUTURAS DE CONCRETO ARMADO EM AMBIENTE MARINHO}




\subsection{TIPOS DE CIMENTOS, RELAÇÃO a/c E CURA}

Estudos apontam que uma maior quantidade de fases aluminato no cimento $\left(C_{3} \mathrm{~A}\right.$ e $\mathrm{C}_{4} \mathrm{AF}$ ) reflete em uma maior fixação química de cloretos, reduzindo assim a quantidade de cloretos livres na solução dos poros do concreto que são passiveis de se movimentar e atingir a região das armaduras (Pereira et al., 2013; Meira et al., 2014, Maes e De Belie, 2014).

Tratando de forma específica a respeito dos cimentos brasileiros, Meira et al. (2007) realizaram um estudo a respeito da penetração de cloretos em concretos expostos em zona de atmosfera marinha à 10 metros de distância do mar por 18 meses de exposição. Foi utilizado o mesmo traço para os concretos, porém com dois tipos de cimento com quantidades distintas de $\mathrm{C}_{3} \mathrm{~A}$. Os resultados mostraram que os concretos executados com o cimento com maior quantidade de $\mathrm{C}_{3} \mathrm{~A}$ acabaram por apresentar a menor frente de penetração de cloretos. Os autores creditam esta ocorrência aos efeitos de fixação química mais pronunciáveis no caso do cimento com maior quantidade de $\mathrm{C}_{3} \mathrm{~A}$.

A respeito da relação água/cimento $(a / c)$, a literatura relaciona este parâmetro à porosidade do concreto, tendo em vista que o aumento na relação a/c eleva a porosidade do concreto e facilita o ingresso de cloretos (Meira et al., 2007; Angst et al., 2009). Os estudos de Meira et al. (2010) e Cheewaket et al. (2012) mostram que quanto maior o valor correspondente a relação $a / c$, maior a concentração de cloretos em uma dada profundidade no concreto. Além disso, Castro et al. (2001) pontuam que maior será a profundidade do pico máximo de penetração de cloretos quanto maior a relação a/c.

Segundo Song et al. (2008) e Meira et al. (2014), além da relação a/c, a cura do concreto também está relacionada à porosidade do mesmo, regulando assim a disponibilidade de água e oxigênio para ocorrência do processo corrosivo, além da facilidade de penetração do agente agressivo. Pereira et al. (2013) e Arya et al. (2014) pontuam que quanto maior o período de cura, menor a absorção de água devido à melhora propiciada pela microestrutura do concreto.

\subsection{ADIÇÕES}

As adições mais conhecidas e estudadas visando à melhora no desempenho do concreto sob aspectos como resistência mecânica e durabilidade são: a cinza volante, a sílica ativa, o metacaulim e a escória de alto forno. Estas, apesar de suas características próprias, podem aumentar a fixação de cloretos e/ou reduzir a permeabilidade do concreto (Papadakis, 2000; Angst et al., 2009; Shi et al., 2012; Camacho et al., 2014).

Sobre a cinza volante, Cheewaket et al. (2012), Shi et al. (2012) e Camacho et al. (2014) pontuam que este é um dos produtos obtidos a partir da combustão de carvão mineral para a produção de energia elétrica, sendo seu uso no concreto recorrente em várias partes do mundo. Segundos os autores, pesquisas na literatura apontam os efeitos positivos da adição de cinza volante através da maior capacidade de fixação química de cloretos devido a grande quantidade de aluminatos em sua composição.

Com relação à sílica ativa, esta é produto da manufatura de ligas ferro-silício. A sílica por sua vez tem como propriedade um refinamento de poros na microestrutura do concreto reduzindo a penetração dos cloretos (Shi et al., 2012; Camacho et al., 2014), além de ser uma adição bastante reativa, capaz de reagir com o hidróxido de cálcio presente na microestrutura do concreto para gerar gel $\mathrm{C}-\mathrm{S}-\mathrm{H}$, um produto que contribui para a maior resistência à compressão do concreto.

O metacaulim e a sílica ativa consomem a portlandita e refinam a microestrutura de poros do concreto, além de aumentar a resistência na zona de interface agregado-matriz (Shi et al., 2012, Wang et al., 2014). Badogiannis et al. (2015) demonstram que a incorporação de metacaulim diminui a quantidade de cloretos totais no concreto em todas as profundidades, quando exposto aos cloretos, comparado à um concreto sem essa adição. Tal fato se deve à diminuição do coeficiente de difusão dos cloretos ocasionado pelo metacaulim.

Os efeitos da escória de alto forno também se manifestam também através do 
refinamento de poros e do aumento na capacidade de fixação química de cloretos devido à presença de aluminatos (Shi et al., 2012, Maes\& De Belie, 2014; Camacho et al., 2014).

\section{ESTUDO DE CASO}

$\mathrm{O}$ estudo de caso abordado neste artigo está localizado na Ilha dos Arvoredos, localizada no litoral sul de São Paulo, na cidade de Guarujá. Estruturas de concreto foram construídas nesta ilha na década de 50. Trata-se de elementos estruturais de concreto presentes em diferentes zonas de agressividade marinha sob condições naturais de degradação, desde a sua construção. A Figura 2 apresenta a estrutura e os diferentes pontos de coleta de material ( $A, B$ e $C$ ) e as diferentes zonas de agressividade marinha onde os elementos estruturais se encontram. No ponto A é possível observar um elemento estrutural de concreto construído junto à zona de variação de maré em meio às formações rochosas da Ilha. Este elemento foi construído para realizar o escoramento das fôrmas para a construção dos demais elementos estruturais. No ponto $B$, o ponto de coleta de material foi realizado no terço inferior do pilar central da estrutura, estando está região presente em zona de respingos. No ponto $C$, por sua vez, o ponto de coleta de material foi a face voltada para o mar da laje da estrutura, haja vistas que este elemento estrutural, além de estar voltado para o mar sem obstáculos à sua frente, não entra em contato com a água do mar, estando assim em zona de atmosfera marinha. Portanto, foram escolhidos pontos pertencentes à estrutura de forma que cada um estivesse localizado em uma zona de agressividade diferente, possibilitando a análise da influência de cada zona em uma estrutura de concreto armado.

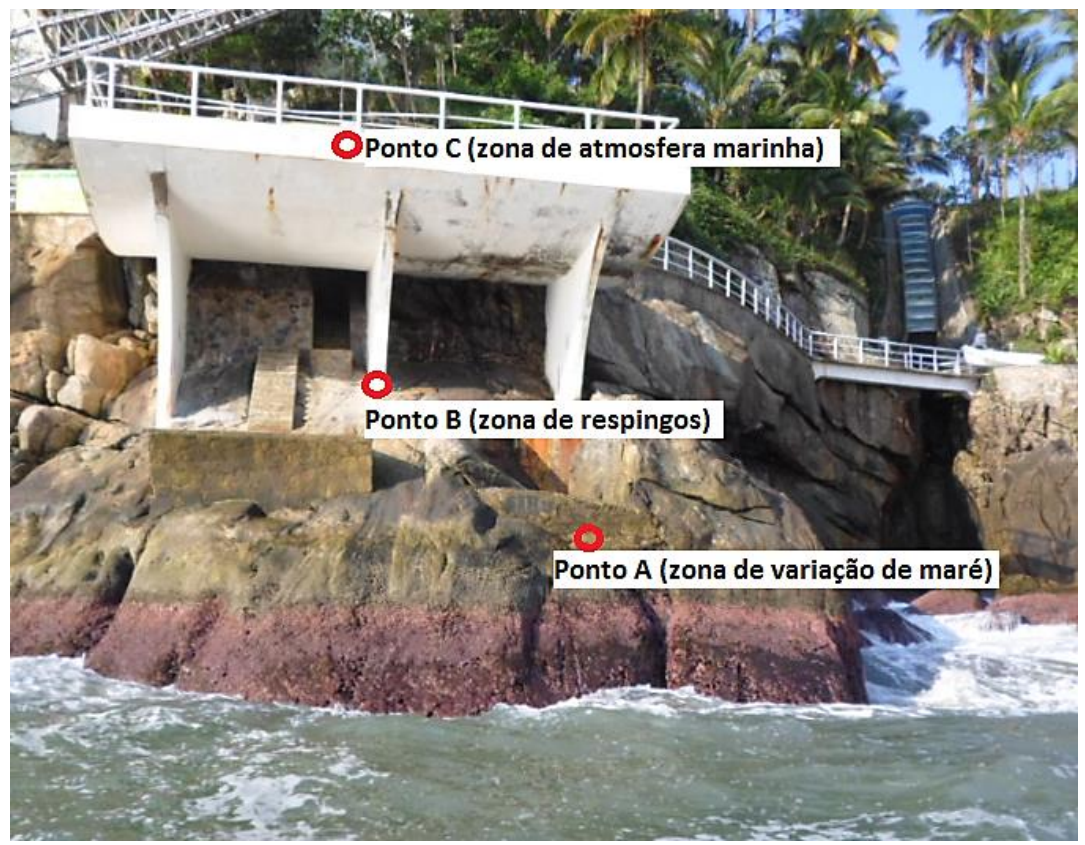

FIGURA 2: Diferentes pontos de coleta de material em estruturas presentes em diferentes zonas de agressividade marinha na llha dos Arvoredos.

FONTE: Autores.

\subsection{METODOLOGIA}

Nesse estudo de caso será abordada a concentração de cloretos em relação à massa de concreto dos pontos A, B e C por meio da espectroscopia de fluorescência de Raios-X. A espectroscopia bombardeia o material de estudo por um feixe de radiação de alta energia. Isso faz com que o material absorva fótons e excite os elétrons, e quando excitados há emissão de luz caracterizando a fluorescência. As amostras utilizadas para análise de fluorescência de Raios- $X$ foram provenientes do pó de concreto retirado das estruturas a uma profundidade de $20 \mathrm{~mm}$.

Em se tratando de normas para reger a 
determinação da concentração de cloretos em amostras de concreto, diversos instrumentos normativos, como por exemplo, a ASTM C1152, ASTM C 1218 e RILEM TC 178-TMC, tratam de técnicas analíticas para a determinação da concentração de cloretos através de procedimentos que envolvem a digestão química da amostra de concreto. Neste caso, existe a possibilidade de volatilização de uma parcela dos cloretos presentes na amostra de concreto, o que não é considerado uma situação ideal. Desta forma, para evitar esse tipo de ocorrência, a determinação de cloretos por meio de espectroscopia de fluorescência de Raios- $X$ foi a metodologia escolhida para esta pesquisa. Esta é uma técnica recente, sem normalização vigente no momento. Contudo, os trabalhos de Torres-Luque et al. (2014), Moradllo, Hu e Ley (2017) e Moradllo et al. (2017) são referência mais recentes a partir da aplicação desta técnica para a determinação de cloretos em amostras de concreto e foram usados como base para este artigo. Essa técnica evita a perda de uma parcela de cloretos, pois não existe volatilização no processo.

\subsection{RESULTADOS: ENSAIOS DE ESPECTROSCOPIA DE FLUORESCÊNCIA DE RAIS-X}

A Figura 3 apresenta a concentração de cloretos totais em relação à massa de concreto determinado por espectroscopia de fluorescência de Raios-X nos pontos A, B e C (Figura 2), onde é possivel observar que as maiores concentrações foram encontradas na zona de variação de maré (A). Além disso, observa-se que a concentração de cloretos totais em relação à massa de concreto varia consideravelmente de acordo com a zona de agressividade marinha, sendo a maior concentração evidenciada na zona de variação de maré $(3,143 \%)$, seguida da zona de respingos $(2,318 \%)$ e, após, da zona de atmosfera marinha $(0,646 \%)$, todas elas em relação à massa de concreto e sujeitas a mesma direção dos ventos e insolação.

Comparando os valores obtidos acerca da concentração de cloretos através da técnica de espectroscopia de fluorescência de Raios- $X$ apresentados na Figura 3 com o valor limite de 0,05 \% em relação à massa de concreto proposto pelo CEB-FIB Bulletin D'Information no 183 (1992) para a ocorrência da despassivação das armaduras, é possível observar que em todos os casos os valores obtidos foram muito acima da concentração limite citada, demonstrando que estes elementos estruturais são candidatos a sofrer com os efeitos da corrosão das armaduras.

Além disso, a técnica de espectroscopia de fluorescência de Raios-X mostrou ser uma técnica eficaz e precisa na determinação da concentração de cloretos em estruturas de concreto em ambiente marinho, onde é possível obter resultados com precisão de três casas decimais em um tempo de análise de aproximadamente 15 minutos, sem provocar a digestão química da amostra.

Os resultados atestam que a zona de variação de maré é a mais agressiva às estruturas de concreto sob a óptica da corrosão das armaduras, tendo em vista a grande concentração de cloretos totais observados, concordando com outros estudos (Ghods et al, 2005; Safehian et al. 2013; Medeiros-Junior et al, 2015a). Os ciclos de molhagem e secagem aos quais a zona de variação de maré está sujeita contribuem para acelerar a penetração dos cloretos no concreto, além de fornecer uma maior fonte de cloretos por estar mais próxima do contato com a água do mar. 


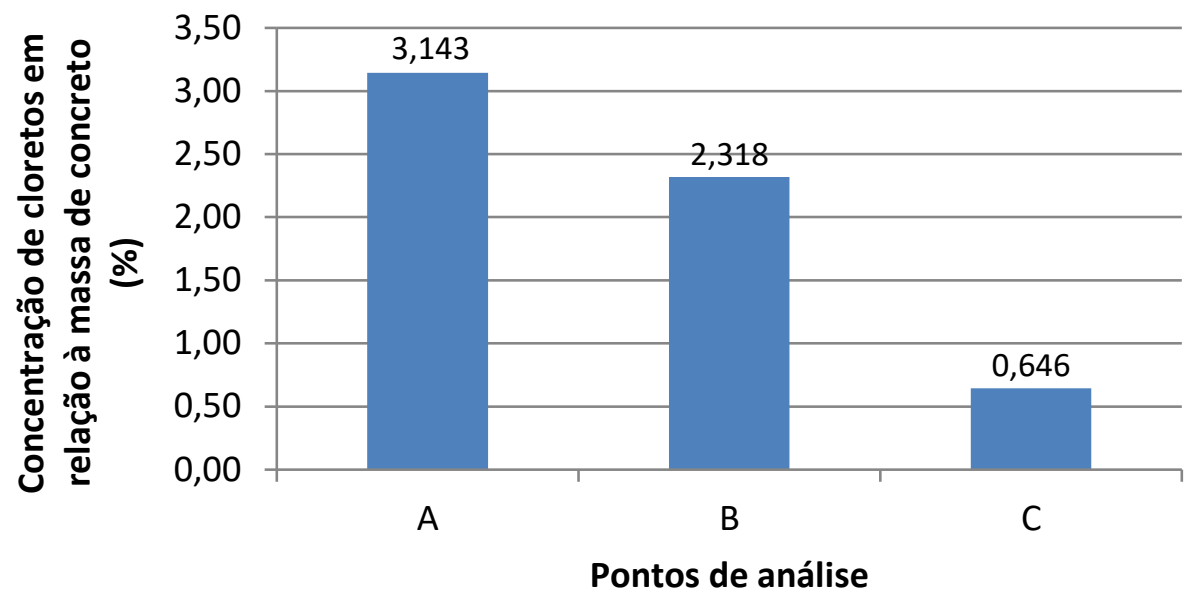

FIGURA 3: Cloretos totais (\%) em relação à massa de concreto determinados em estruturas presentes em diferentes zonas de agressividade marinha.

FONTE: Autoria própria.

\section{CONCLUSÃO}

1. Embora vários estudos sejam conduzidos para tratar da corrosão em ambiente marinho, a degradação de elementos estruturais continua sendo recorrente em diversas obras de infraestrutura. Tal fato está associado em primeiro lugar a uma premissa de projeto, onde é admitido que a camada de cobrimento de concreto é suficientemente capaz de resistir ao ingresso de cloretos. Isto, aliado à falta de planos de monitoramento e manutenção, contribui para a degradação precoce destas estruturas. Destaca-se que os cobrimentos propostos pela norma brasileira consideram apenas o macroclima (com algumas distinções para a zona marinha). Dessa forma, esse artigo levanta a importância de mais estudos que permitam uma proposta de cobrimentos por microclima, ou a partir de modelos de previsão que incorporem esses efeitos de uma maneira mais adequada, devido a quantidade de fatores ambientais que afetam a penetração de cloretos, conforme discutido neste artigo, além do Brasil ser um país continental com ambientes marinhos em condições ambientais ( $T, U R$, ventos) diferentes.

2. O conhecimento das variáveis que tangem a penetração de íons cloreto em estruturas de concreto armado é uma ferramenta importante a fim de determinar o tempo para que os cloretos atinjam a região das armaduras e desencadeiem um processo corrosivo, entretanto, muitos são os parâmetros ambientais e materiais envolvidos. Desta forma, modelar a penetração de cloretos em estruturas de concreto apresenta-se como uma árdua tarefa que requer estudos regionalizados sobre o tema.

3. De acordo com o tipo de zona marinha onde as estruturas podem estar presentes, o nível de agressividade quanto à corrosão induzida por cloretos é variável. Isto pode ser observado através do estudo de caso realizado neste artigo, onde diferentes concentrações de cloretos foram identificadas por espectroscopia de fluorescência de Raios-X em amostras de concreto coletadas em pontos presentes em diferentes zonas de agressividade marinha. A zona de variação de maré se mostrou a mais agressiva sob a perspectiva da corrosão das armaduras.

\section{AGRADECIMENTOS}

Os autores agradecem à Coordenação de Aperfeiçoamento de Pessoal de Nível Superior (CAPES) pelo fomento à pesquisa. 


\section{REFERÊNCIAS BIBLIOGRÁFICAS}

AMERICAN SOCIETY FOR TESTING AND MATERIALS, ASTM C1152: Standard Test Method for Acid-Soluble Chloride in Mortar and Concrete. West Conshohocken, United States of America, 2012.

ASTM C1218: Standard Test Method for WaterSoluble Chloride in Mortar and Concrete. West Conshohocken, United States of America, 2008.

ASTM G140: Standard Test Method for Determining Atmospheric Chloride Deposition Rate by Wet Candle Method. West Conshohocken, United States of America, 2008.

ANGST, U. et al. Critical Chloride Content in Reinforced Concrete - A Review. Cement and Concrete Research, Vol. 39, 2009, 1122-1138 p.

APOSTOLOPOULOS, C. A. et al. Chloride-induced corrosion of steel reinforcement - Mechanical performance and pit depth analysis. Construction and Building Materials, Vol. 38, 2013, 139-146 p.

ARYA, C. et al. Chloride penetration in concrete subject to wet-dry cycling: influence of moisture content. Structures and Building, Vol. 167, n. SB2, 2014, 94-107 p.

BACKUS, J. et al. Exposure of mortars to cyclic chloride ingress and carbonation. Advances in Cement Research, Vol. 25, n. 1, 2013, 3-11 p.

BADOGIANNIS, E. et al. Evaluation of chloridepenetration resistance of metakaolin concrete by means of a diffusion - Binding model and of the k-value concept. Cement and Concrete Composites, Vol. 63, 2015, 1-7 p.

BALESTRA, C. E. T. Influência do Grau de Corrosão na Resistência à Tração de Armaduras - Estudo de Caso das Fundações da Nova Ala Zero do ITA. Dissertação (Mestrado em Infraestrutura Aeronáutica) - Instituto Tecnológico de Aeronáutica. São José dos Campos, SP, 2013, 163 p.

BASTIDAS-ARTEAGA, E. et al. Influence of weather and global warming in chloride ingress into concrete: $A$ stochastic approach. Structural Safety, Vol. 32, 2010, 238-249 p.

BROOMFIELD , J. P. Corrosion of Steel in Concrete Understanding, Investigation and Repair. Taylor \& Francis, London and New York, 2007, 294 p.

CAMACHO, J. B. et al. The influence of ion chloride on concretes made with sulfate-resistant cements and mineral admixtures. Construction and Building Materials, Vol. 70, 2014, 483-493 p.

CASCUDO, O. O Controle da Corroão de Armaduras em Concreto - Inspeção e Técnicas Eletroquímicas. Editora Pini, São Paulo, 1998, 254p.
CASTRO, P. et al. Interpretation of chloride profile from concrete exposed to tropical marine environments. Cement and Concrete Research, Vol. 31, 2001, 529-537 p.

CHEEWAKET, T. et al. Initial corrosion presented by chloride threshold penetration of concrete up to 10 year results under marine site. Construction and Building Materials, Vol. 37, 2012, 693-698 p.

COMITÉ EURO-INTERNATIONAL DU BÉTON, Bulletin D'Information, n. 183: Durable concrete structures. CEBFIP model code 1990 design code. Lausanne, 1992. 112 p.

Bulletin D'Information $\mathrm{n}$ ㅇ192: Diagnosis and assessment of concrete structures. Lausanne, Thomas Telford, 1989, 130p.

CORVO, F. et al. The influence of airborne salinity on the atmospheric corrosion of steel. Corrosion Science, Vol. 37, n. 12, 1995, 1889-1901 p.

DA COSTA, A. et al. Modelling of chloride penetration into non-saturated concrete: case study application for real marine offshore structures. Construction and Building Materials, Vol. 43, 2013, 217-224 p.

FRAÇOIS, R. et al. Impact of corrosion on mechanical properties of steel embedded in 27-year-old corroded reinforced concrete beams. Material and Structures, Vol. $46,2013,889-910 p$.

GHODS P. et al. The effect of different exposure conditions on the chloride diffusion into concrete in the Persian Gulf Region. Proceeding of the 3rd International Conference on Construction Materials on Performance, Innovations and Structural Implications. Vancouver, Canadá, 2005.

HAN, S. J. et al. Degradation of Flexural Strength in Reinforced Concrete Members Caused by Steel Corrosion. Construction and Building Materials, Vol. 54, 2014, 572-583 p.

JAEGERMANN, C. Effect of water-cement ratio and curing on chloride penetration into concrete exposed to mediterranean sea climate. ACl Materials Journal, Vol. 97, n. 4, 1990, 333-339 p.

KHAN, I. et al. Prediction of Reinforcement Corrosion Using Corrosion Induced Cracks Width in Corroded Reinforced Concrete Beams. Construction and Building Materials, Vol. 56, 2014, 84-96 p.

KUOSA, H. et al. Effect of coupled deterioration by freeze-thaw, carbonation and chlorides on concrete service life. Cement and Concrete Composites, Vol. 47, 2014, 32-40 p.

LEE, J. S.; MOON, H. Y. Salinity Distribution of Seashore Concrete Structures in Korea - Building and Environment. Vol. 41, 2006, 1447-1453 p. 
LI, L. et al. Numerical simulation of chloride penetration in concrete in rapid chloride migration tests. Cement and Concrete Composites, Vol. 63, 2015, 113-121 p.

LINDVALL, A. Chloride ingress data from field and laboratory exposure - Influence of salinity and temperature. Cement and Concrete Composites, Vol. 29, 2007, 88-93 p.

LIU, R. et al. Influence of carbonation on chlorideinduced reinforcement corrosion in simulated concrete pore solutions. Construction and Building Materials, Vol. 56, 2014, 16-20 p.

MAES, M.; DE BELIE, N. Resistance of concrete and mortar against combined attack of chloride and sodium sulphate. Cement and Concrete Composites, Vol. 53, 2014, 59-72 p.

MAZER, W. Metodologia para a previsão da penetração de íons cloreto em estruturas de concreto armado utilizando lógica difusa. Tese (Doutorado em Física e Química dos Materiais Aeroespaciais) - Instituto Tecnológico de Aeronáutica. São José dos Campos, SP, 2009, 237 p.

MEDEIROS M. H. F. et al. Reinforced Concrete in Marine Environment: Effect of Wetting and Drying Cycles, Height and Positioning in Relation to the Sea. Construction and Building Materials, Vol. 44, 2013, 452$457 \mathrm{p}$.

MEDEIROS-JUNIOR R. A. et al. Chloride penetration into concrete in an offshore platform-analysis of exposure conditions. Ocean Engineering, Vol. 103, 2015a, 78-87 p.

MEDEIROS-JUNIOR R. A. et al. Service life of concrete structures considering the effects of temperature and relative humidity on chloride transport. Environment, Development and Sustainability.,Vol. 17, 2015b, 1103$1119 \mathrm{p}$.

MEHTA, P. K.; MONTEIRO, P. J. M. Concreto microestrutura, propriedades e materiais. Editora IBRACON, 10 edição em português traduzida da 3o edição em inglês, São Paulo, 2008, 674p.

MEIRA, G. R. et al. Chloride Penetration into Concrete Structures in the Marine Atmosphere Zone Relationship Between Deposition of Chlorides on the Wet Candle and Chlorides Accumulated into Concrete. Cement \& Concrete Composites, Vol. 29, 2006, 667-676 p.

MEIRA, G. R. et al. Salinity of marine aerosol in a Brazilian coastal area - Influence of wind regime. Atmospheric environment, Vol. 41, 2007, 8431-8441 p.

MEIRA, G. R. et al. Durability of Concrete Structures in Marine Atmosphere Zones - The Use of Chloride Deposition Rate on the Wet Candle as na Environmental Indicator. Cement \& Concrete composites, Vol. 32, 2010, 427-435 p.
MEIRA, G. R. et al. Analysis of chloride threshold from laboratory and field experiments in marine atmosphere zone. Construction and Building Materials, Vol. 55, 2014, 289-298 p.

MORADLLO, M.K. et al. Using X-ray fluorescence to image chlorides profiles in concrete. Cement and Concrete Research, Vol. 92, 2017, 128-141 p.

MORADLLO, M. K.; HU, Q.; LEY, M. T. Using X-ray imaging to investigate in-situ ion diffusion in cementitious materials. Construction and Building Materials, Vol. 136, 2017, 88-98 p.

MORCILLO, M. et al. Salinity in marine atmospheric corrosion: its dependence on the wind regime existing in the site. Corrosion Science, Vol. 42, 2000, 91-104 p.

MUSTAFA, M. A.; YUSOF, K. M. Atmospheric chloride penetration into concrete in semi tropical marine environment. Cement and Concrete Research, Vol. 24, n. 4, 1994, 661-670 p.

$\mathrm{OH}$, B. H.; JANG, S. Y. Effect of material and environmental parameters on chloride penetration profiles in concrete structures. Cement and Concrete Research, Vol. 37, 2007, 47-53 p.

PAPADAKIS, V. G. Effect of supplementary cementing materials on concrete resistance against carbonation and chloride ingress. Cement and Concrete Research, Vol. 30, 2000, 291-299 p.

PEREIRA, V. C. O. et al. Influence of cement type in reinforcement corrosion of mortars under action of chlorides. Construction and Building Materials, Vol. 40, 2013, 710-718 $p$

PONTES, R. B. Disseminação de íons cloreto na orla marítima do bairro de Boa Viagem, Recife-PE. Dissertação (mestrado em engenharia civil) Universidade Católica de Pernambuco. Recife, PE, 2006. $121 \mathrm{p}$.

RILEM RECOMMENDATION TC 178-TMC. Testing and Modeling Chloride Penetration in Concrete - Analysis of Total Chloride Content in Concrete. Materials and Structures, Vol. 35, 2002, 583-585 p.

ROMANO, F. S. Estudo do ingresso de cloretos em concretos localizados no Litoral Norte do Rio Grande do Sul. Dissertação (mestrado) - Universidade Federal do Rio Grande do Sul. Escola de Engenharia. Porto Alegre, RS, 2009, 153 p.

SAFEHIAN, M. et al. Assessment of service life models for determination of chloride penetration into silica fume concrete in the severe marine environmental condition. Construction and Building Materials, Vol. 48, 2013, 287$294 \mathrm{p}$. 
SAILLIO, M. et al. Chloride binding in sound and carbonated cementitious materials with various types of binder. Construction and Building Materials, Vol. 68, 2014, 82-91 p.

SAMARAKOON, S. M. S. M.; RATNAYAKE, R. M. Residual service life prediction of offshore concrete structures with chloride-induced damage: the state of the art. Proceedings of the ASME, $32^{\text {nd }}$ International Conference on Ocean, Offshore and Arctic Engineering, 2013.

$\mathrm{SHI}$ X. et al. Durability of steel reinforced concrete in chloride environments: an overview. Construction and Building Materials, Vol. 30, 2012, 125-138 p.

SONG, H. W. et al. Factors influencing chloride transport in concrete structures exposed to marine environments. Cement and Concrete Composites, Vol. 30, 2008, 113-121 p.

TORRES-LUQUE, M. et al. Non-destructive methods for measuring chloride ingress into concrete: State-of-theart and future challenges. Construction and Building Materials, Vol. 68, 2014, 68-81 p.

TUUTTI, K. Corrosion of Steel in Concrete. Stokholm, Swedish Cement and Concrete Reseach, Fo n. 504, 1982.

WANG, G. M. et al. Experimental investigation on chloride diffusion and binding in concrete containing metakaolin. Corrosion Engineering, Science and Technology, Vol. 49, 2013, 282-286 p.

WANG, Z. et al. Corrosion of rebar in concrete under cyclic freeze-thaw and chloride salt action. Construction and Building Materials, Vol. 53, 2014, 40-47 p.

WU, J. et al. Transport model of chloride ions in concrete under loads and drying-wetting cycles. Construction and Building Materials, Vol. 112, 2016, 733-738 p.

YUAN, Q. et al. Chloride binding of cement based materials subjected to external chloride environment a review. Construction and Building Materials, Vol. 23, 2009, 1-13 p.

ZHU, W. et al. Effect of Corrosion of Reinforcement on the Mechanical Behavior of Highly Corroded RC Beams. Construction and Building Materials, Vol. 56, 2013, 544$554 \mathrm{p}$.

$\mathrm{ZHU}, \mathrm{W}$. et al. Experimental Investigation of the Relationship Between Residual Cross-Section Shapes and the Ductility of Corroded Bars. Construction and Building Materials, Vol. 69, 2014, 335-345 p. 